\title{
Biomedical applications of plasmon resonant metal nanoparticles
}

\author{
Hongne Liao', \\ Collen L Neh'2 \& \\ Jason H Hafiner ${ }^{1,2 \dagger}$ \\ ${ }^{\dagger}$ Author for correspondence \\ 1 D epartment of Chemistry, \\ Rice U niversity, H ouston, \\ TX, USA \\ 2D epartment of Physics \& \\ Astronomy, Rice U niversity, \\ H ouston, TX, USA \\ Tel.: +1 713348 3205; \\ Fax: +1 713348 4150; \\ E-mail: hafner@rice.edu
}

\begin{abstract}
The strong optical absorption and scattering of noble metal nanoparticles is due to an effect called localized surface plasmon resonance, which enables the development of novel biomedical applications. The resonant extinction, which can be tuned to the near-infrared, allows the nanoparticles to act as molecular contrast agents in a spectral region where tissue is relatively transparent. The localized heating due to resonant absorption, also tunable into the near-infared, enables new thermal ablation therapies and drug delivery mechanisms. The sensitivity of these resonances to their environment leads to simple affinity sensors for the detection of low-level molecular analytes. Coupled with their general lack of toxicity, these applications suggest that noble metal nanoparticles are a highly promising class of nanomaterials for new biomedical applications.
\end{abstract}

\section{Keywords}

bionanotechnology, biosensing, nanoparticle, nanophotonics, optical coherence tomography, optoacoustic tomography, photothermal therapy, plasmonics, plasmon resonance, thermal ablation therapy, thermolytic therapy

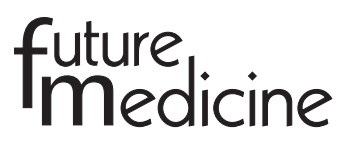

An intensive research effort over the past 20 years has yielded nanoparticles of controlled size and structure that are composed of a variety of materials, including semiconductors, metals, oxides, fullerenes and organics. These nanomaterials often exhibit novel, tunable physical properties not found in their molecular or bulk precursors. When coupled to surface ligands for a controlled interface with their environment, the nanoparticle properties enable the development of new biomedical technologies for the detection and treatment of disease [1-5]. Common themes include their use as molecular imaging contrast agents, sensors for disease markers, vehicles for drug delivery and therapeutic agents based on their novel properties. Each class of nanomaterial has a unique set of properties that can be exploited in different ways. In this review, we will outline several biomedical applications of noble metal nanoparticles (hereafter referred to simply as 'nanoparticles') that are made possible owing to localized surface plasmon resonance (LSPR).

Localized surface plasmon resonance Localized surface plasmon resonance occurs when an electromagnetic field drives the collective oscillations of a nanoparticle's free electrons into resonance. This essentially classical effect was described theoretically by M ie in 1908 by solving $M$ axwell's equations for a metal sphere surrounded by a dielectric medium using the dielectric function of the bulk metal [6]. The dielectric function of gold and silver yields resonances at visible wavelengths for spherical nanoparticles [7]. The collective electron oscillations cause extinction of incident light by two mechanisms: Rayleigh scattering of the light into other directions and absorption of the light to generate heat. Therefore, gold and silver nanoparticle solutions exhibit strong peaks in their extinction spectra at visible wavelengths. $\mathrm{N}$ anoparticles with diameters less than approximately $1 / 10$ the radiation wavelength are excited as simple dipole oscillators. D ipole-limited nanoparticles are not strong scatterers, thus their extinction is dominated by absorption with resonance linewidths of less than $100 \mathrm{~nm}[8,9]$. As the nanoparticle diameter increases beyond the dipole limit, higher-order excitations become significant, which greatly increase the scattering efficiency and broaden the resonance since they peak at longer wavelengths.

Although the basic LSPR phenomenon has been long understood, the field has experienced significant growth recently owing to advances in the synthesis of nanoparticles with more complex shapes and structures [10]. As aforementioned, spherical nanoparticles are only tunable by increasing their size beyond the dipole limit. For example, gold nanoparticles of $20 \mathrm{~nm}$ in diameter have a resonance at a wavelength of $520 \mathrm{~nm}$ in water, which red shifts to $600 \mathrm{~nm}$ owing to broadening as the nanoparticle diameter increases to $100 \mathrm{~nm}$. Beyond this size, the resonance continues to shift but becomes significantly broadened [11]. Silver nanoparticle solutions are known to exhibit multicolored plasmon resonances, although the varied wavelengths are actually due to nonspherical shapes that exist in typical silver colloid preparations [8]. The key to achieving broad tunability without sacrificing 
the linewidth of the resonance is to create nanoparticles with controlled, nonspherical shapes. $\mathrm{N}$ anoparticles whose resonances are tuned into the near-infrared (NIR) spectral region wheretissue is relatively transparent are especially valued for biomedical applications [12]. O ne path to N IR plasmon resonances is simply to aggregate the spherical nanoparticles [13], however aggregates are difficult to fabricate reproducibly. The syntheses of several well defined, monodisperse nanoparticles have been developed, whose resonant wavelength can betuned through the visible and NIR spectrum (Figure 1). M etallodielectric gold nanoshells were used for initial demonstrations of several of the N IR applications described here [14]. M ore recently, surfactant-directed synthesis has enabled the high-yield production of gold nanorods [15,16], which are also tunable [17]. Silver nanoparticles can be synthesized with controlled shape and optical properties guided by optical illumination [18]. M ore complex architectures have also been described, including gold nanocages [19-21], elongated core-shell geometries [22] and gold nanostars [23-28].

\section{Biomedical imaging contrast}

The plasmon resonant scattering from a single gold or silver nanoparticle is many orders of magnitudebrighter than the signal from single fluorophores, fluorescent beads or quantum dots in microscopic imaging applications [29,30]. In addition, the scattering signal does not photobleach or blink and polarization reveals the local orientation for nonspherical nanoparticles [31]. These effects have generated interest in the use of gold and silver nanoparticles as microscopic imaging labels [32]. However, the large nanoparticle size

\section{Figure 1. Transmission electron micrographs of}

goldnanoparticles with localized surface plasmon resonance that can be tuned through the visible and near-infrared.
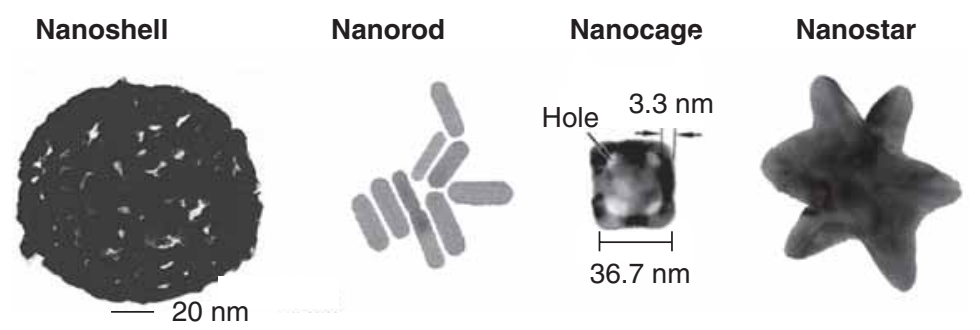

The nanoparticles are all shown to the same scale.

Adapted from [14] (nanoshell), [19] (nanocage) and [26] (nanostar). The nanorod image is supplied by the authors. relative to fluorophores and quantum dots may limit intracellular imaging applications. Resonant scattering applications are therefore more likely to focus on particle tracking experiments and molecular contrast in tissues [33]. For example, the two-photon luminescence from NIR resonant gold nanorods has been used to monitor microscopic blood flow in vivo (Figre2) [34]. Single particle imaging has also been exploited in a release assay to sense the functional activity of a biomolecular target, as opposed to simply measuring its concentration [35].

Fiber-based endoscopic imaging modalities provide high resolution in vivo imaging of tissue microanatomy with contrast based on changes in the refractive index [36]. Potential imaging mechanisms include optical coherence tomography (OCT), reflectance confocal microscopy (RCM) and optoacoustic tomography [37]. While these 'optical biopsies' achieve a resolution sufficient for the early diagnosis of disease based on cellular anatomy, their contrast is not ideally suited for imaging specific molecular changes. Imaging contrast and molecular specificity can be greatly enhanced by plasmon resonant scattering or absorption from targeted noble metal nanoparticles. Gold nanoparticles conjugated to anti-epidermal growth factor receptor (EGFR) have been used to target epithelial precancers specifically and provide imaging contrast sensitive to the EG FR expression level due to particle agglutination [38]. Nonspherical nanoparticles have also been pursued as contrast agents. Their N IR resonances could enable investigation deeper into tissue and even the possibility of diagnostic imaging with exogenous illumination and detection. Bioconjugated gold nanoshells [39-41], gold nanocages [19,21] and gold nanorods [42] are being developed for this purpose. These nanoparticles have been conjugated to specifically target cancer cells in vitro, where their specificity has been confirmed by dark-field optical microscopy (Figre3). N anoshells and nanocages have also been demonstrated to provide enhanced contrast OCT in vitro [19,41] and gold nanospheres and nanorods have enhanced optoacoustic tomography [37].

Drug delivery \& thermal ablation therapy Plasmon resonant absorption of light by noble metal nanoparticles provides a nanoscopic heat source and enables novel therapeutic strategies for the treatment of disease. In a manner similar to photodynamic therapy [43], plasmon resonant 


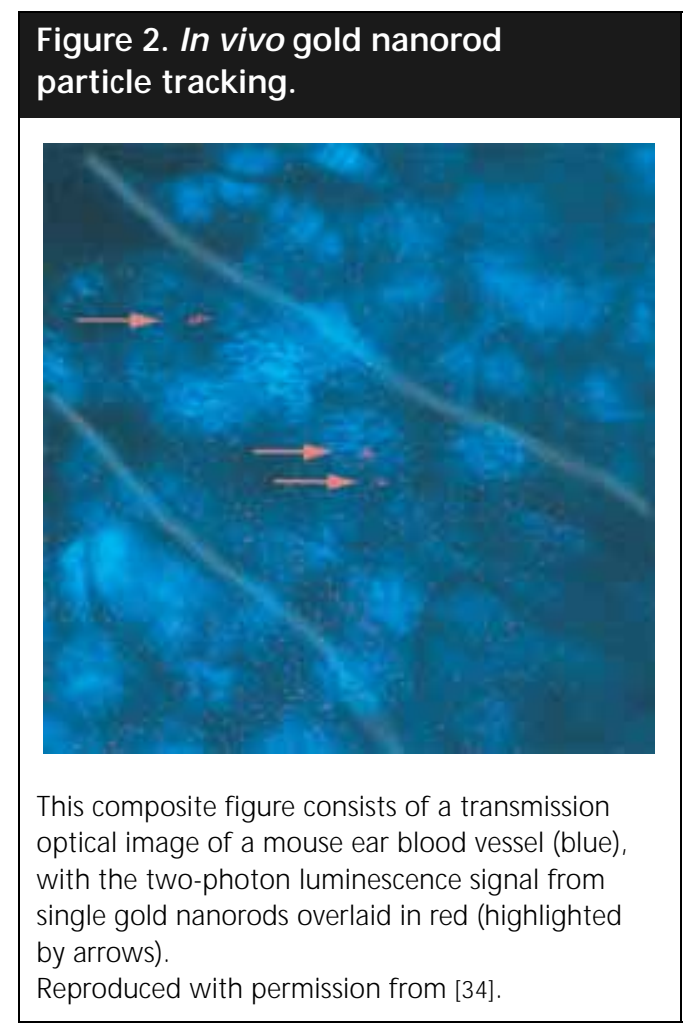

nanoparticles can be delivered systemically and activated locally by exposure to resonant illumination. The local temperature increase can deliver drugs bound with the nanoparticle or have a direct photothermal or thermolytic therapeutic effect. For example, gold nanoshell-hydrogel composite materials have been loaded with protein and then illuminated at the plasmon resonance to stimulate release by
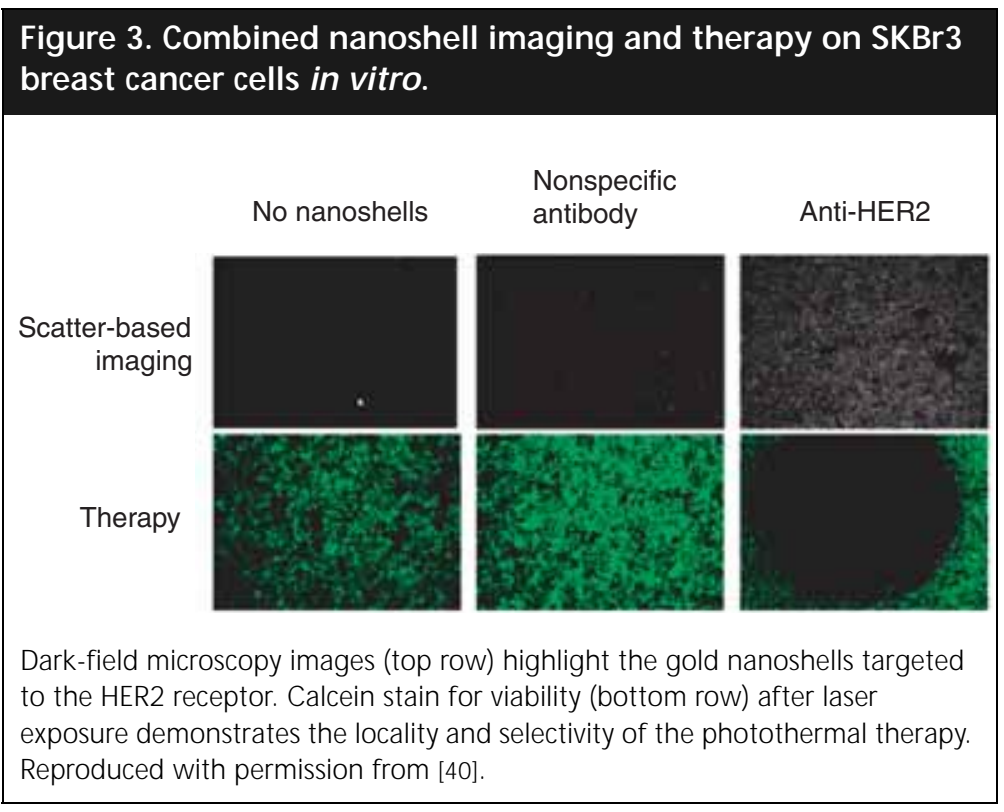

shrinking the hydrogel [44]. The nanoshells caused enhanced drug release and enabled multiple bursts of protein by modulated heating. Such devices could have significant drug delivery applications, especially if the stimulant radiation can be administered exogenously to NIR resonant nanoparticles. A similar strategy has been pursued based on hollow polymer capsules filled with the substances to be delivered [45-47]. The capsule walls were impregnated with gold or silver nanoparticles so that absorption of light damaged the capsules, thus releasing their contents. D elivery has also been accomplished by associating the drug directly with the nanoparticle. D N A has been bound to lipid-stabilized gold nanorods. U pon resonant illumination, the nanorods transformed into spheres and the DNA was released without significant structural degradation, based on gel electrophoresis [48]. H owever, the function of the released genetic material has not yet been confirmed.

Thermal ablation treatments for cancer rely on the local application of heat to destroy diseased tissue selectively. Thermal therapies are simple and minimally invasive relative to conventional surgical treatments, although their effectiveness is limited by the ability to locally and specifically apply heat so as not to destroy healthy tissue. Plasmon resonant nanoparticles are highly effective enhancers of thermal ablation therapies since they can heat tissue locally owing to resonant absorption of radiation and can be targeted to tumors. Gold nanoshells photothermally destroyed breast carcinoma cells in vitro with a continuous wave (CW) NIR laser radiation dose that did not harm cells in the absence of the nanoparticles (Figure3) [40]. Similar results were reported recently using conjugated gold nanorods [42]. An in vivo study in mice relied on the relatively high permeability of tumor vasculature to target the nanoparticles. Gold nanoshells were administered intravenously and deposited at the tumor site. Exogenous radiation of the tumor with NIR resonant radiation caused abatement of the tumor with excellent survival of the treated mice [49].

Excitation with pulsed lasers further enhances the degree to which nanoparticles locally heat the target tissue since less time is available for the heat to diffuse from the nanoparticle. Gold nanoparticles of $30 \mathrm{~nm}$ in diameter have been conjugated to $C D 8^{+} T$ lymphocytes and exposed to $20 \mathrm{~ns}, 565 \mathrm{~nm}$ pulses in vitro, resulting in selective loss of viability in the targeted cell population [50]. Pulsed excitation has also been explored 
to selectively purge leukemic cells from a cell suspension. Gold nanoparticles of $30 \mathrm{~nm}$ in diameter were targeted to the leukemic cells and made to form clusters through the use of primary and secondary monoclonal antibodies. Upon pulsed laser excitation, these clusters form microbubbles owing to rapid heating at their surface, locally destroying the target cells thermolytically [51]. These experiments demonstrate that pulsed laser excitation can localize thermal ablation therapies to the cellular level.

\section{Biosensing applications}

The plasmon resonances of noble metal nanoparticles are sensitive to the dielectric properties of their local environment. This effect has been exploited for a range of sensing strategies in which the presence of the molecule to be detected alters the extinction spectrum. In fact, one of the earliest demonstrations of nanoparticle biosensing relied on this effect $[52,53]$. G old nanoparticles were functionalized with two-probe oligonucleotide sequences and exposed to a target oligonucleotide sequence, whose two ends were complementary to the probe sequences. When mixed, the target and probes hybridized, causing aggregation of the gold nanoparticles. The unhybridized gold nanoparticle solutions were red in color, typical of gold nanoparticles whose plasmon resonant peak absorption is $520 \mathrm{~nm}$. Upon aggregation, the plasmon resonances became severely damped, resulting in a blue color and eventually precipitation of the colloid. This dramatic change in spectral properties can be detected colorimetrically, yielding an extremely simple means of sensing specific oligonucleotide sequences, an application of considerable biomedical interest. Since this initial demonstration, several significant extensions of the technique have been developed to detect single oligonucleotide base pair mismatches [54], sequence amplified D N A [55] and reduce the limits of detection drastically [56]. These advances are the result of chemical properties of the gold nanoparticles, such as their high density of D N A coverage and their ability to reduce silver to enhance the signals [57].

Analyte-induced nanoparticle aggregation can also be applied to protein and small molecule sensing, a concept that is certainly not new [58]. H owever, several advances in nanoparticle synthesis and conjugation have brought new capabilities to aggregation assays with plasmon resonant nanoparticles. H eterobifunctional cross-linkers provide nanoparticle bioconjugates that are more stable under physiological conditions and are more resistant to fouling. For example, a poly(ethylene) glycol linker has been developed with aldehyde distal to the nanoparticle surface. The aldehyde was functionalized with lactose to create a reversible and specific aggregation sensor sensitive to lectins [59]. The advent of plasmon resonant nanoparticles tunable to the N IR has enabled detection in optically turbid media. Using gold nanoshells, immunoglobulins were detected in whole blood below $\mathrm{ng} / \mathrm{ml}$ concentrations [60]. Recently, aptamers have been applied as the selective binding ele ment rather than antibodies. With gold nanoparticles conjugated to aptamers for plateletderived growth factor (PD GF), different aptamerbinding strengths to different PDGF isoforms could be distinguished and a competitive assay to detect PD GF receptors was demonstrated [61].

As an alternative to the drastic optical changes due to nanoparticle aggregation, one can monitor the more subtle effect of the binding of a target molecule to the nanoparticle surface [62-66]. The presence of the target molecule alters the local dielectric environment of the nanoparticle, which shifts the LSPR peak wavelength. Since interparticle interactions are not required, the nanoparticles can be supported on a solid substrate to avoid aggregation and, therefore, improve stability of the sensor. When the nanoparticles are coupled to antibodies or aptamers for specificity, LSPR sensors represent a very simple and potentially inexpensive strategy for sensing low-level, label-free analytes in complex media. LSPR sensing with nanoscopic arrays of silver triangles created by nanosphere lithography has proven highly effective $[67,68]$. W ith such substrates, amyloid- $\beta$-derived diffusible ligands (ADD Ls), a biomarker for Alzheimer's disease, have been detected at 10-pM concentrations (Figure 4) [69]. Biomedical applications, including a comparison of LSPR and SPR sensing techniques [70], have been reviewed thoroughly [71,72].

Table 1 reviews the LSPR sensing properties of several gold and silver nanostructures, evaluated by analyzing their spectra in media with different indices of refraction. The sensitivity is reported as 'nm/RIU ' or 'eV/RIU', meaning the shift in LSPR peak wavelength or photon energy per unit change of refractive index. The range of observed sensitivity values demonstrates a strong dependence on the nanoparticle shape and composition. It has been highlighted recently that the plasmon resonant linewidth should also be considered in measurements of sensitivity, since the linewidth will affect the ultimate detectivity of an LSPR sensor [73]. A unitless figure of merit (FOM) was therefore cal culated, in which the sensitivity values 
Figure 4. Localized surface plasmon resonance sensing of amyloid-derived diffusible ligands. The titration demonstrates detectivity dow $\mathrm{n}$ to $10 \mathrm{pM}$.
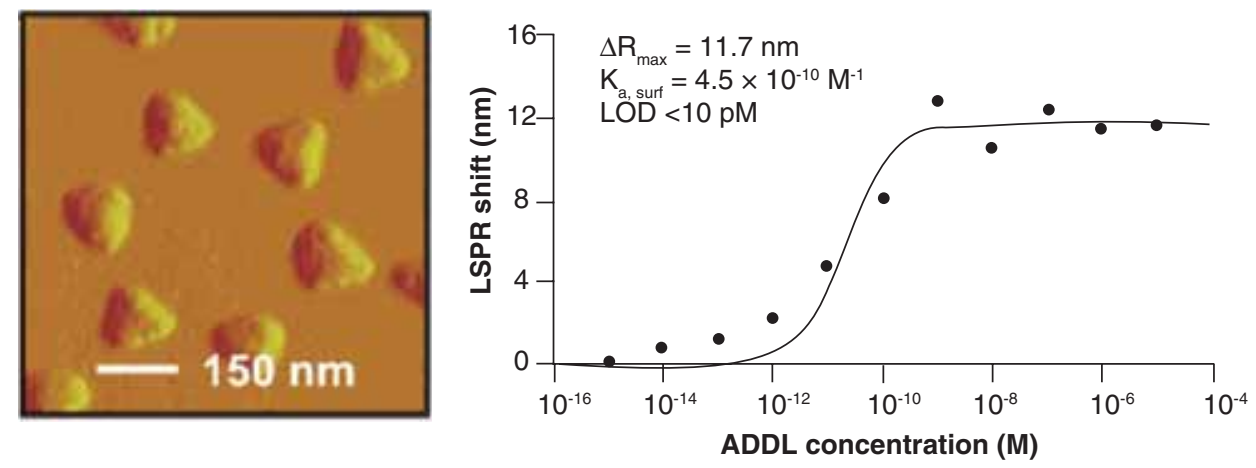

The sensor substrate consists of nanoscopic silver triangles.

$\Delta R_{\text {max }}$ : M aximum shift; ADDL: Amyloid- $\beta$-derived diffusible ligands; $K_{a, \text { surf }}$ : Association constant; LOD: Limit of detection; LSPR: Localized surface plasmon resonance.

Reproduced with permission from [69].

described were divided by the resonance linewidth. According to this FOM, silver nanocubes, silver nanotriangles and gold nanostars are the most highly sensitive nanoparticles. Although the core-shell nanoparticles in Tablel have the highest $\mathrm{nm} / \mathrm{RIU}$ shifts, their FOM is low owing to the broad, low energy resonances exhibited by these nanoparticles. However, since grating-based instruments disperse light linear with wavelength and since some applications may be limited by spectral resolution rather than the signal-to-noise ratio, the nm/RIU shift could be the most significant parameter for certain sensing applications. The ideal nanoparticle for LSPR sensing will be strongly application dependent.

\section{Conclusions}

LSPR in noble metal nanoparticles results in strong visible and N IR scattering and absorption, which enables significant biomedical applications. Resonant scattering and absorption provide molecular contrast agents for biomedical imaging and resonant absorption enables the development of novel drug delivery methods as well as

Table 1. LSPR properties reported for several gold and silver nanostructures.

\begin{tabular}{|c|c|c|c|c|c|c|c|c|c|c|}
\hline \multirow[t]{2}{*}{ Author } & \multirow[t]{2}{*}{ Particle } & \multirow{2}{*}{$\begin{array}{l}\text { Single/ } \\
\text { ensemble }\end{array}$} & \multicolumn{2}{|c|}{ Resonance } & \multicolumn{2}{|c|}{ Linewidth } & \multicolumn{2}{|c|}{ Shift/RIU } & \multirow[t]{2}{*}{ FOM } & \multirow[t]{2}{*}{ Ref. } \\
\hline & & & $\mathrm{nm}$ & eV & $\mathrm{nm}$ & meV & $\mathrm{nm}$ & meV & & \\
\hline Tam (2004) & $\mathrm{Au} / \mathrm{SiO}_{2}$ shell & Ensemble & 770 & 1.61 & 350 & 732 & 314 & 657 & 0.9 & [76] \\
\hline Sun (2002) & Au/AuS shell & Ensemble & 700 & 1.77 & 400 & 1012 & 409 & 1035 & 1.0 & [77] \\
\hline Wang (2006) & Au nanorice & Ensemble & 1600 & 0.775 & 600 & 291 & 801 & 388 & 1.3 & [22] \\
\hline Underwood (1994) & Au sphere & Ensemble & 530 & 2.34 & 060 & 265 & 090 & 397 & 1.5 & [78] \\
\hline Raschke (2004) & Au/AuS shell & Single & 660 & 1.88 & 077 & 220 & 117 & 333 & 1.5 & [79] \\
\hline Sherry (2005) & Ag cube & Single & 510 & 2.43 & 091 & 433 & 146 & 695 & 1.6 & [73] \\
\hline M alinsky (2001) & Ag triangle & Ensemble & 564 & 2.20 & 104 & 405 & 191 & 745 & 1.8 & [62] \\
\hline Nehl (2006) & Au star & Single & 675 & 1.84 & 125 & 340 & 238 & 649 & 1.9 & [26] \\
\hline M ock (2003) & Ag sphere & Single & 520 & 2.38 & 073 & 335 & 160 & 734 & 2.2 & [80] \\
\hline M acFarland (2003) & Ag particle & Single & 585 & 2.12 & 49 & 178 & 203 & 736 & 4.1 & [81] \\
\hline M ock (2003) & Ag triangle & Single & 760 & 1.63 & 080 & 172 & 350 & 751 & 4.4 & [80] \\
\hline Nehl (2006) & Au star & Single & 770 & 1.61 & 124 & 260 & 665 & 1410 & 5.4 & [26] \\
\hline Sherry (2005) & Ag cube-sub & Single & 430 & 2.88 & 022 & 146 & 118 & 792 & 5.4 & [73] \\
\hline
\end{tabular}

To compare the figures of merit, results are only shown for reports that provide either the resonance linewidth or LSPR spectrum. FOM: Figure of merit; LSPR: Localized surface plasmon resonance; meV: Millielectron volt; RIU: Refractive index unit. 
photothermal and thermolytic laser therapies. These applications can be carried out with exoge nous illumination with N IR resonant nanoparticles. The sensitivity of plasmon resonances to their local environment enables sensing schemes, which provide simple, label-free mechanisms to transduce molecular binding in specific affinity assays. The broad array of applications already demonstrated, the pace of development in nanoparticle synthesis and the general low toxicity of noble metal nanoparticles suggests that LSPR resonances will have a significant impact on medical applications of nanotechnology [74].

\section{Future perspectives}

$M$ any challenges remain before the applications described here can be brought into clinical use. Although new nanoparticle structures are synthesized constantly, the exact mechanisms of their syntheses are rarely understood. N ew methods of studying complex nanoparticle synthesis reactions are needed to unravel these mechanisms. The use of commercially available heterobifunctional cross-linkers has had, and will continue to have, a positive impact on biological applications of noble metal nanoparticles. $\mathrm{H}$ owever, their effectiveness in vivo may be reduced. $\mathrm{N}$ ew targeting strategies, such as one demonstrated recently based on an adenoviral vector, should be pursued actively [75]. Also, standardized, quantitative assays for bioconjugated nanoparticles are required to determine their activity over biological applications. While these open questions affect the general development of biomedical applications of plasmon resonant nanoparticles, it appears likely that some of the demonstrations described here will reach clinical use in the next 10 years.

\section{Executive summary}

\section{Plasmon resonant nanoparticles}

- Collective excitations of free electrons in gold and silver nanoparticles leads to resonant optical absorption and scattering in the visible and near-infrared (NIR).

- The resonance wavelength can be tuned through the visible and NIR with nanoparticle shape, and resonant linewidths are typically 10 s to 100 s of nanometers.

- Scattering cross sections are many orders larger than fluorophores and quantum dots, and the signal does not bleach or blink.

\section{Biomedical imaging contrast}

- Bioconjugated gold nanoparticles enhance molecular contrast in 'optical biopsies' performed endoscopically.

- NIR resonant nanoparticles demonstrated targeted imaging in vitro and show promise for biomedical imaging applications with exogenous illumination.

\section{Drug delivery and thermal ablation therapies}

- Resonant absorption creates a local heat source that can be used for the targeted and controlled release of drugs associated with the nanoparticles.

- Resonant absorption can have direct photothermal or thermolytic therapeutic effects on tumors, as demonstrated in vivo and at the cellular level.

\section{Biosensing applications}

- Sensitivity of the plasmon resonance to the local environment yields simple, label-free mechanisms to transduce molecular binding in affinity assays.

\section{Bibliography}

1. Salata OV: Applications of nanoparticles in biology and medicine. J. N anobiotechnology 2, 1-6 (2003).

2. Englebienne $\mathrm{P}$, Van $\mathrm{H}$ oonacker $\mathrm{A}$ : Bionanotechnology: the science of revealing life with nanostructures. Curr. N anosci. 1, 97-106 (2005).

3. Roco M C: N anotechnology: convergence with modern biology and medicine. Curr. O pin. Biotechnol. 14, 337-346 (2003).

4. Portney N G, O zkan M : N ano-oncology: drug delivery, imaging, and sensing. Anal. Bioanal. Chem. 384, 620-630 (2006).
5. Hernano A, Crespo P, G arcia M A: M etallic magnetic nanoparticles. Scientific World J. 5 , 972-1001 (2005).

6. M ie G : Articles on the optical characteristics of turbid tubes, especially colloidal metal solutions. Annalen Physik 25, 377-445 (1908).

7. Johnson PB, Christy RW: O ptical constants of noble metals. Phys Rev. B 6, 4370-4379 (1972).

8. M ock JJ, Barbic M, Smith D R et al.: Shape effects in plasmon resonance of individual colloidal silver nanoparticles. J. Chem. Phys. 116, 6755-6759 (2002).
9. Link S, El-Sayed M A: Size and temperature dependence of the plasmon absorption of colloidal gold nanoparticles. J. Phys. Chem. B 103, 4212-4217 (1999).

10. Murphy CJ, San TK, Gole AM et al.: Anisotropic metal nanoparticles: synthesis, assembly, and optical applications. J. Phys. Chem. B 109, 13857-13870 (2005).

11. Link S, El-Sayed M A: Shape and size dependence of radiative, non-radiative and photothermal properties of gold nanocrystals. Int. Rev. Phys. Chem. 19, 409-453 (2000). 


\section{Biomedical applications of plasmon resonant metal nanoparticles - REV IEW}

12. Weissleder $\mathrm{R}: \mathrm{A}$ clearer vision for in vivo imaging. $N$ at. Biotechnol. 19, 316-317 (2001).

13. Khlebtsov N G, M elnikov AG, D ykman LA et al.: O ptical properties and biomedical applications of nanostructures based on gold and silver bioconjugates. In: Photopolarimetry in Remote Sensing. Videem G, Yatskiv YS, M ishchenko M I (Eds). Kluwer, The N etherlands 265-308 (2004).

14. O Idenburg SJ, Averitt RD, Westcott SL et al.: $N$ anoengineering of optical resonances. Chem. Phys. Lett. 288, 243-247 (1998).

15. Sau TK, M urphy CJ: Seeded high yield synthesis of short $\mathrm{Au}$ nanorods in aqueous solution. Langmuir 20, 6414-6420 (2004).

16. Nikoobakht B, El-Sayed M A: Preparation and growth mechanism of gold nanorods (N Rs) using seed-mediated growth method. Chem. M ater. 15, 1957-1962 (2003)

17. Link S, M ohamed M, El-Sayed M : Simulation of the optical absorption spectra of gold nanorods as a function of their aspect ratio and the effect of the medium dielectric constant. J. Phys. Chem. B 103, 3073-3077 (1999).

18. M aillard $M, H$ uang $P R$, Brus L: Silver nanodisk growth by surface plasmon enhanced photoreduction of adsorbed $[\mathrm{Ag}+]$. $N$ ano Lett. 3, 1611-1615 (2003).

19. Chen J, Saeki F, Wiley B et al.: Gold nanocages: bioconjugation and their potential use as optical imaging contrast agents. N ano Lett. 5, 473-477 (2005).

20. Chen JY, Wiley B, Li ZY \& al.: Gold nanocages: engineering their structure for biomedical applications. Adv. M ater. 17, 2255-2261 (2005).

21. Cang H , Sun T, Li ZY et al.: G old nanocages as contrast agents for spectroscopic optical coherence tomography. 0 pt. Lett. 30, 3048-3050 (2005).

22. Wang $\mathrm{H}$, Brandl DW, LeF et al.: $\mathrm{N}$ anorice: a hybrid plasmonic nanostructure. $\mathrm{N}$ ano $\mathrm{Lett}$. 6, 827-832 (2006).

23. Yamamoto M , Kahiwagi Y, Sakata T et al.: Synthesis and morpohology of star-shaped gold nanoplates protected by poly( $\mathrm{N}$-vinyl-2pyrrolidone). Chem. $M$ ater. 17, 5391-5393 (2005).

24. Sau TK, M urphy CJ: Room temperature, high-yield synthesis of multiple shapes of gold nanoparticles in aqueous solution. J. Am. Chem. Soc. 126, 8648-8649 (2004).

25. H ao E, Bailey R, Schatz G et al.: Synthesis and optical properties of "branched" gold nanocrystals. N ano Lett. 4, 327-330 (2004).

26. N ehl CL, Liao H W, H afner JH : O ptical properties of star-shaped gold nanoparticles. N ano Lett. 6, 683-688 (2006).
27. Chen S, Wang Z, Ballato J et al.: M onopod, bipod, tripod, and tetrapod gold nanocrystals. J. Am. Chem. Soc. 125, 16186-16187 (2003).

28. Burt JL, Elechiguerra JL, Reyes-G asga J et al.: Beyond Archimedean solids: star polyhedral gold nanocrystals. J. Cryst. Growth 285, 681-691 (2005).

29. Yguerabide J, Yguerabide EE: Lightscattering submicroscopic particles as highly fluorescent analogs and their use as tracer labels in clinical and biological applications - II. Experimental characterization. Anal. Biochem. 262, 157-176 (1998).

30. Schultz S, Smith DR, M ock JJ et al.: Single target molecule detection with nonbleaching multicolor optical immunolabels. Proc. Natl Acad. Sci. USA 97, 996-1001 (2000).

31. Sönnichsen C, Alivisatos AP: Gold nanorods as novel nonbleaching plasmonbased orientation sensors for polarized single-particle microscopy. N ano Lett. 5, 301-304 (2005).

32. Schultz D A: Plasmon resonant particles for biological detection. Curr. 0 pin. Biotechnol. 14, 13-22 (2003).

33. Boppart $\mathrm{SA}, \mathrm{O}$ Idenburg $\mathrm{AL}, \mathrm{Xu} \mathrm{CY}$ et al.: $O$ ptical probes and techniques for molecular contrast enhancement in coherence imaging. J. Biomed. 0 pt. 10, 1-14 (2005).

34. Wang $\mathrm{H}, \mathrm{H}$ uff $\mathrm{TB}, \mathrm{Z}$ weifel $\mathrm{DA}$ et al.: In vitro and in vivo two-photon luminescence imaging of single gold nanorods. Proc. N atl Acad. Sci. U SA 102, 15752-15756 (2005).

35. Glass JR, Dickerson JC, Schultz D A: Enzyme-mediated individual nanoparticle release assay. Anal. Biochem. 353, 209-216 (2006).

36. Tearney GJ, Brezinkski M E, Bouma BE et al.: In vivo endoscopic optical biopsy with optical coherence tomography. Science 276, 2037-2039 (1997).

37. Copland JA, Eghtedari M , Popov VL et al.: Bioconjugated gold nanoparticles as a molecular based contrast agent: implications for imaging of deep tumors using optoacoustic tomography. M ol. Imaging Biol. 6, 341-349 (2004).

38. Sokolov K, Follen M, Aaron J et al.: Realtime vital optical imaging of precancer using anti-epidermal growth factor receptor antibodies conjugated to gold nanoparticles. Cancer Res. 63, 1999-2004 (2003).

39. Loo C, H irsch L, Lee M H et al.: Gold nanoshell bioconjugates for molecular imaging in living cells. 0 pt. L ett. 30, 1012-1014 (2005).
40. Loo C, Lowery $\mathrm{A}, \mathrm{H}$ alas $\mathrm{N}$ et al.: Immunotargeted nanoshells for integrated cancer imaging and therapy. $\mathrm{N}$ ano Lett. 5, 709-711 (2005).

41. Loo C, Lin A, H irsch $L$ et al.: N anoshellenabled photonics-based imaging and therapy of cancer. Technol. Cancer Res. Treat. 3, 33-40 (2004).

42. H uang X, El-Sayed IH, Q ian W et al.: Cancer cell imaging and photothermal therapy in the near-infrared by using gold nanorods. J. Am. Chem. Soc. 128, 2115-2120 (2006).

43. H uang Z: A review of progress in clinical photodynamic therapy. Technol. Cancer Res Treat. 4, 283-293 (2005).

44. Sershen SR, Westcott SL, H alas NJ et al.: Temperature-sensitive polymer-nanoshell composites for photothermally modulated drug delivery. J. Biomed. M ater. Res. 51, 293-298 (2000).

45. Skirtach AG, Antipov AA, Shchukin DG et al.: Remote activation of capsules containing ag nanoparticles and ir dye by laser light. Langmuir 20, 6988 (2004).

46. Angelatos AS, Radt B, C aruso F: Lightresponsive polyelectrolyte/gold nanoparticle microcapsules. J. Phys. Chem. B 109, 3071-3076 (2005).

47. Radt B, Smith TA, Caruso F: O ptically addressable nanostructures capsules. Adv. M ater. 16, 2184-2189 (2004).

48. Takahashi H, Niidome Y, Yamada S: Controlled release of plasmid D N A from gold nanorods induced by pulsed nearinfrared light. Chem. Commun. 2005, 2247-2249 (2005).

49. $O$ ' $N$ eal $D P, H$ irsch $L R, H$ alas $N$ J et al.: Photo-thermal tumor ablation in mice using near infrared-absorbing nanoparticles. Cancer Lett. 209, 171-176 (2004).

50. Pitsillides $C M$, Joe EK, Wei XB et al.: Selective cell targeting with light-absorbing microparticles and nanoparticles. Biophys. J. 84, 4023-4032 (2003).

51. Lapotko D, Lukianova E, Potapnev M et al.: M ethod of laser activated nanothermolysis for elimination of tumor cells. Cancer Lett. 239, 36- 45 (2006).

52. Elghanian R, Storhoff JJ, Mucic RC et al.: Selective colorimetric detection of polynucleotides based on the distancedependent optical properties of gold nanoparticles. Science 277, 1078-1081 (1997).

53. M irkin $C A$, Letsinger $R L$, M ucic RC et al.: A D N A-based method for rationally assembling nanoparticles into macroscopic materials. N ature 382, 607-609 (1996). 
54. Storhoff JJ, Elghanian R, M ucic RC et al.: $O$ ne pot colorimetric differentiation of polynucleotides with single base imperfections using gold nanoparticle probes. J. Am. Chem. Soc. 120, 1959-1964 (1998).

55. Li H X, Rothberg LJ: Label-free colorimetric detection of specific sequences in genomic DN A amplified by the polymerase chain reaction. J. Am. Chem. Soc. 126, 10958-10961 (2004).

56. Rosi N L, M irkin CA: N anostructures in biodiagnostics. Chem. Rev. 105, 1547-1562 (2005).

57. M orrissey JH : Silver stain for proteins in polyacrylamide gels - a modified procedure with enhanced uniform sensitivity. Anal. Biochem. 117, 307-310 (1981).

58. Leuvering JHW, Thal PJHM , Vanderwaart $M$ et al.: A sol particle agglutination assay for human chorionicgonadotropin. J. Immunol. M ethods 45, 183-194 (1981).

59. O tsuka H, Akiyama $Y, N$ agasaki $Y$ et al.: $Q$ uantitative and reversible lectin-induced association of gold nanoparticles modified with $\alpha$-lactosyl- $\omega$-mercapto-poly(ethylene glycol). J. Am. Chem. Soc. 123, 8226-8230 (2001).

60. H irsch LR, Jackson JB, LeeA et al.: A whole blood immunoassay using gold nanoshells. Anal. Chem. 75, 2377-2381 (2003).

61. Huang $\mathrm{CC}, \mathrm{H}$ uang $\mathrm{YF}, \mathrm{C}$ ao $\mathrm{ZH}$ et al.: Aptamer-modified gold nanoparticles for colorimetric determination of plateletderived growth factors and their receptors. Anal. Chem. 77, 5735-5741 (2005).

62. M alinsky M D, K elly KL, Schatz GC et al.: $C$ hain length dependence and sensing capabilities of the localized surface plasmon resonance of silver nanoparticles chemically modified with alkanethiol self-assembled monolayers. J. Am. Chem. Soc. 123, 1471-1482 (2001).
63. Englebienne P: U se of colloidal gold surface plasmon resonance peak shift to infer affinity constants from the interactions between protein antigens and antibodies specific for single or multiple epitopes. Analyst 123, 1599-1603 (1998).

64. Nath N, Chilkoti A: A colorimetric gold nanoparticle sensor to interrogate biomolecular interactions in real time on a surface. Anal. Chem. 74, 504-509 (2002).

65. Endo $\mathrm{T}, \mathrm{Kerman} \mathrm{K}, \mathrm{N}$ agatani $\mathrm{N}$ et al.: Label-free detection of peptide nucleic acidD N A hybridization using localized surface plasmon resonance based optical biosensor. Anal. Chem. 77, 6976-6984 (2005).

66. Kalele SA, Ashtaputre SS, H ebalkar N Y et al.: 0 ptical detection of antibody using silica-silver core-shell particles. Chem. Phys. Lett. 404, 136-141 (2005).

67. Hulteen JC, Van D uyne RP: N anosphere lithography - a materials general fabrication process for periodic particle array surfaces. J. Vac. Sci. Technol. A 13, 1553-1558 (1995).

68. H aes AJ, Van D uyne RP: A nanoscale optical blosensor: sensitivity and selectivity of an approach based on the localized surface plasmon resonance spectroscopy of triangular silver nanoparticles. J. Am. Chem. Soc. 124, 10596-10604 (2002).

69. H aes AJ, Chang L, Klein WL et al.: $D$ etection of a biomarker for Alzheimer's disease from synthetic and clinical samples using a nanoscale optical biosensor. J. Am. Chem. Soc. 127, 2264-2271 (2005).

70. Yonzon $C R$, Jeoungf $E$, Zou SL et al.: A comparative analysis of localized and propagating surface plasmon resonance sensors: the binding of concanavalin A to a monosaccharide functionalized selfassembled monolayer. J. Am. Chem. Soc. 126, 12669-12676 (2004).

71. H aes AJ, Van D uyne RP: A unified view of propagating and localized surface plasmon resonance biosensors. Anal. Bioanal. Chem. 379, 920-930 (2004).
72. H aes AJ, Van D uyne RP: Preliminary studies and potential applications of localized surface plasmon resonance spectroscopy in medical diagnostics. Expert Rev. M ol. Diagn. 4, 527-537 (2004).

73. Sherry LJ, Chang S-H, Schatz GC et al.: Localized surface plasmon resonance spectroscopy of single silver nanocubes. Nano Lett. 5, 2034-2038 (2005).

74. Connor E, M wamukaJ, G ole A et al.: Gold nanoparticles are taken up by human cells but do not cause acute cytotoxicity. Small 1 , 325- 327 (2005).

75. Everts M, Saini V, Leddon JL et al.: Covalently linked au nanoparticles to a viral vector: potential for combined photothermal and gene cancer therapy. Nano Lett. 6, 587-591 (2006).

76. Tam F, M oran C, H alas N : G eometrical parameters controlling sensitivity of nanoshell plasmon resonances to changes in dielectric environment. J. Phys. Chem. B 108, 17290-17294 (2004).

77. Sun Y, Xia Y: Increased sensitivity of surface plasmon resonance of gold nanoshells compared to that of gold solid colloids in response to environmental changes. Anal. Chem. 74, 5297-5305 (2002).

78. Underwood S, M ulvaney P: Effect of the solution refractive index on the colors of gold colloids. Langmuir 10, 3427-3430 (1994).

79. Raschke G, Brogl S, Susha AS et al.: G old nanoshells improve single nanoparticle molecular sensors. $N$ ano Lett. 4, 1853-1857 (2004).

80. M ock JJ, Smith DR, Schultz S: Local refractive index dependence of plasmon resonance spectra from individual nanoparticles. N ano L ett. 3, 485-491 (2003).

81. M CFarland AD, Van D uyneRP: Singlesilver nanoparticles as real-time optical sensors with zeptomole sensitivity. $\mathrm{N}$ ano Lett. 3 , 1057-1062 (2003). 